\title{
A better facial contour accomplished by parotid duct preserving superficial parotidectomy
}

Jun Ho Park, Chang Yong Choi, Syeo Young Wee, Young Man Lee

Department of Plastic and Reconstructive Surgery, Soonchunhyang University Gumi Hospital, Gumi, Korea

\begin{abstract}
Depression of facial contour after parotidectomy is still challenging to many of surgeons. A 68-year-old man presented with a 4-month history of a painless swelling in both parotid area. The mass was multiple and fixed at the parotid region. We conducted a parotid duct preserving bilateral superficial parotidectomy by one-stage operation to remove the multiple tumors. A lazy $\mathrm{S}$ incision was made in both preauricular area and the peripheral branches of the facial nerve were identified using surgical landmark. After dissecting the branches of the facial nerve and parotid duct, main parotid duct was preserved but only small fine ductules from the superficial lobe were ligated. Pa $\urcorner$ rotid gland was excised from its anterior aspect with about $1 \mathrm{~cm}$ of normal parotid tissue margin. The patient was followed up for 6 years to evaluate postoperative parotid gland function and the computed tomography (CT) was taken. Patient was satisfied with no sig $\urcorner$ nificant complication such as sunken changes in facial contour, facial nerve function. As far as we know, it is the first study to compare long-term soft tissue contours of soft tissue of duct preserving superficial parotidectomy with duct sacrificing superficial parotidectomy by means of CT findings.
\end{abstract}

Keywords: Parotid duct / Parotid neoplasms / Parotidectomy

\section{INTRODUCTION}

Classically, superficial parotidectomy is most common approach for benign parotid neoplasm such as pleomorphic adenoma and Warthin's tumor. However, frequent complications such as occurrence of Frey's syndrome and depression of facial contour are still challenging to many of surgeons.

Incidence of Frey's syndrome after parotid gland surgery has been reported as wide as $2 \%$ to $96 \%$ [1] and it is various in authors, according to the types of surgical approach. Ogreden et al. [2] compared partial superficial parotidectomy to superficial parotidectomy in terms of Frey's syndrome development and had found no significant difference among them. On the other hand, Papa-

\footnotetext{
Correspondence: Chang Yong Choi

Department of Plastic and Reconstructive surgery, Soonchunhyang University Gumi Hospital, Soonchunhyang University College of Medicine, 179 1gongdan-ro, Gumi 39371, Korea

E-mail: ccysy@hanmail.net

*This work was supported by the Soonchunhyang University Research Fund.

Received November 14, 2017 / Revised February 7, 2018 / Accepted February 21, 2018
}

dogeorgakis et al. [3] reported Frey syndrome ratio among patients they performed partial superficial parotidectomy as 5\%, and among patients who underwent superficial parotidectomy as $18 \%$. Another frequent complication is facial and neck deformity and the cosmetic results may affect quality of life especially in young patients.

We report our clinical experience and outcomes by comparing clinical, photographic, and postoperative computed tomography (CT) findings of parotid duct preserving superficial parotidectomy to conventional superficial parotidectomy.

\section{CASE REPORT}

A 68-year-old man presented with a 4-month history of a painless swelling in both parotid area. The mass was multiple and fixed at the parotid region. He had no medical or operation history. Neither facial palsy nor tumor extension to oropharynx was seen. 
Fine needle aspiration cytology revealed that the lesion was consistent with a few clusters of ductal epithelial cells and stromal cells. An enhanced CT scan showed multiple tumors within bilateral parotid gland. In view of these investigations and clinical presentation at that stage, the presumed diagnosis was the Warthin's tumor.

We conducted a parotid duct preserving bilateral superficial parotidectomy by one-stage operation to remove the multiple tumors. A lazy S incision was made in both preauricular area and the peripheral branches of the facial nerve were identified using surgical landmark (main parotid duct and posterior facial vein without nerve stimulator). After dissecting the branches of the facial nerve and parotid duct, main parotid duct was preserved but only small fine ductules from the superficial lobe were ligated. Parotid gland was excised from its anterior aspect with about $1 \mathrm{~cm}$ of normal parotid tissue margin (Fig. 1). Histological examination of the specimen postoperatively confirmed $5 \times 2 \times 1.5 \mathrm{~cm}$-sized Warthin's tumor in right superficial parotid gland and five segments of Warthin's tumors (1.5 cm in diameter of the largest one) in left superficial parotid gland.

Patient was followed up for 6 years to evaluate postoperative parotid gland function. The preoperative and postoperative photographs with the computed tomography scans were taken (Fig. 2). Patient was satisfied with no significant complication such as sunken changes in facial contour, facial nerve function.

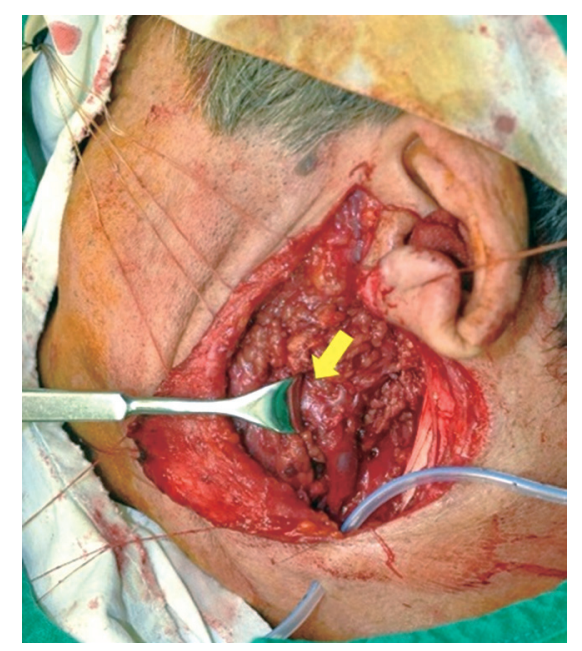

Fig. 1. Intraoperative photographic finding of parotid duct preserving superficial parotidectomy. The main parotid duct (arrow) was preserved after resection of the tumor.
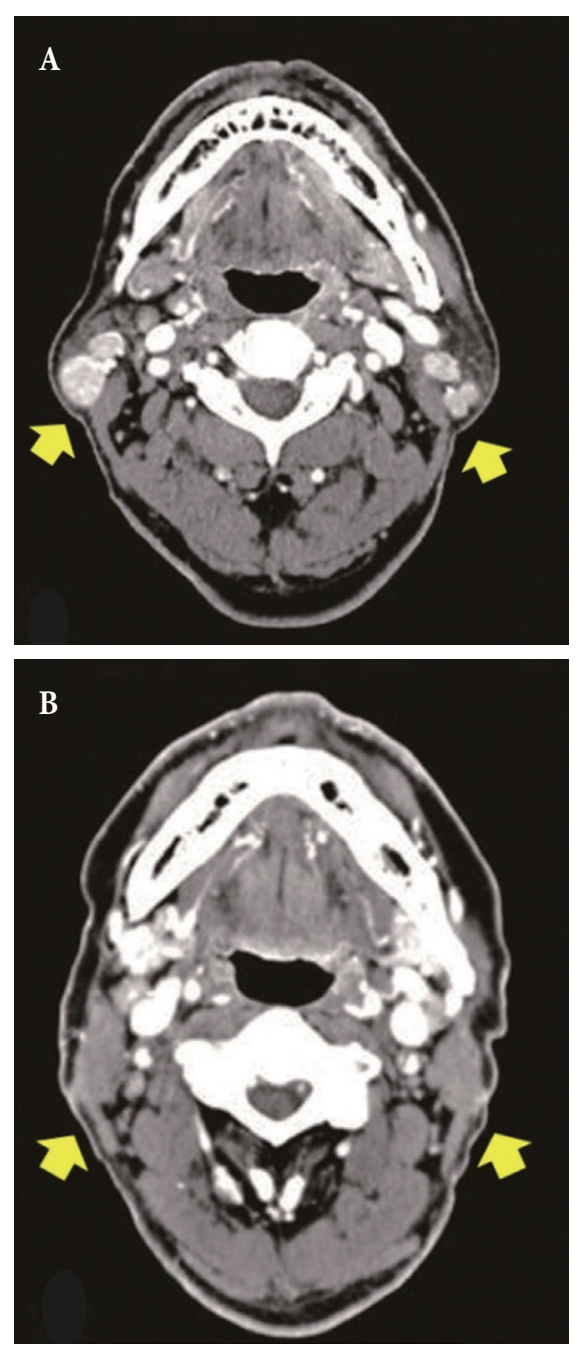

Fig. 2. A 68-year-old male patient with bilateral tumor who underwent parotid duct preserving superficial parotidectomy. (A) Preoperative computed tomography (CT) finding. (B) Postoperative $\mathrm{CT}$ finding in 6 years. Postoperative volumetric changes on both side were minimal (arrows).

However, increased sweating and mild edema on left preauricular area was noticed after 6 months of follow-up period, which was revealed as Frey's syndrome after conducting Minor starch test. At the follow-up date of 6 years, the symptoms of sweating and edema were all gone.

\section{DISCUSSION}

Classically, the main parotid duct was ligated during the superficial parotidectomy or total parotidectomy by reason of easy and 
accurate removal of parotid gland without facial nerve damage and security of the safe margin. In spite of the low recurrence rate, the patients are afflicted with the postoperative complications. Recognized complications or sequelae of parotidectomy were Frey's syndrome and contour deformity where glandular tissue was removed $[4,5]$. However, the parotid duct preserving parotidectomy from our department have achieved both clinical and cosmetic goals in the bilateral multiple tumor cases. It does not interrupt the patency of Stensen's duct and remaining parotid tissues to maintain their function. Preserving the parotid parenchyma and parotid duct helps retaining function of the salivary gland.

Anatomically, the parotid duct passes in an anterior direction superficial to the masseter about $1 \mathrm{~cm}$ below the zygomatic arch and at the anterior border of the masseter, turns in a medial direction to pierce the buccinator and enters the oral cavity at the parotid papilla opposite the second maxillary molar [6]. The detailed anatomy of the parotid duct as received relatively little attention, but in 1956, Davis et al. [7] found that the duct was contributed to by smaller ductules that arose from the superficial and deep lobes. In 2004, Richards et al. [6] described the branching pattern of the parotid duct in cadaver heads. In $31.0 \%$, the parotid duct was seen to pass the deep lobe of the parotid gland where numerous small ductules drained into it. In the remaining ducts, $69.0 \%$ were formed by a branching pattern with more than two major branches. In all cases, the deep lobe of the parotid envelopes the parotid duct with only small ductules connecting the superficial lobe with the parotid duct [6]. This anatomy allows parotid duct preserving partial superficial parotidectomy operation.

Once the peripheral branches of facial nerve are identified and main parotid duct preserving procedure was done, superficial lobe of the parotid gland can be exteriorized by "inter-lobular plane" between superficial and deep lobes without damaging facial nerve. Other then, only isthmus between facial nerve trunk connects superficial lobe and deep lobe. Because there are no nerve fibers in isthmus of parotid, superficial lobe could be dissected from deep lobe and facial nerve by blunt dissection.

After reviewing medical chart from 2007 to 2017 retrospectively, duct preserving surgery was processed in 15 patients whereas, seven patients received conventional duct sacrificing parotidectomy. The indication of the surgery was benign parotid neoplasm such as pleomorphic adenoma or Warthin's tumor localized in superficial parotid duct without further involvement. However, the incidence of Frey's syndrome after surgery was similar comparing the medical chart of both groups. Eleven out of 15 patients (73\%) who underwent duct preserving surgery and five out of seven patients (71\%) who underwent conventional superficial parotidectomy experienced postoperative Frey's syndrome.

Many methods, such as the superficial musculoaponeurotic system advancement flap, fascia lata flap, and sternocleidomastoid muscle flap have been attempted to prevent Frey's syndrome and depressed contour in the past [8]. However, these procedures have the disadvantage of causing donor-site morbidity. To avoid this additional risk, our operative procedure has definite advantage.

After long-term follow-up period of 6 years with duct preserved patients, the surgical region depression was not clearly noticed on photographic and CT findings. On the other hand, the depression was markedly noticed in patient without preserving parotid duct surgery who followed up for 2 years (Fig. 3). Thus, we could conclude that remaining parotid duct prevented atrophy of normal parotid tissues.

The difficulty of this surgery is that only skilled surgeon can try and accomplish this approach without any mistake or unexpected sequalae. Finding the small fine ductule to the superficial lobe is a delicate procedure because of complex anatomy around the parotid gland. Careful dissection and ligation should be done with accurate and delicate handling.

The limitation of our study is that we only included patients who underwent subtotal parotid resection diagnosed of benign parotid tumor such as pleomorphic adenoma and Warthin's tumor. Secondly, the comparison of the outcome of patients with total parotidectomy should be performed in the future. Nevertheless, as far as we know, it is the first study to compare long-term soft tissue contours of soft tissue of duct preserving superficial parotidectomy with duct sacrificing superficial parotidectomy by means of CT findings even though there were a few attempts preserving duct conserving superficial parotidectomy [9]. Statistical 

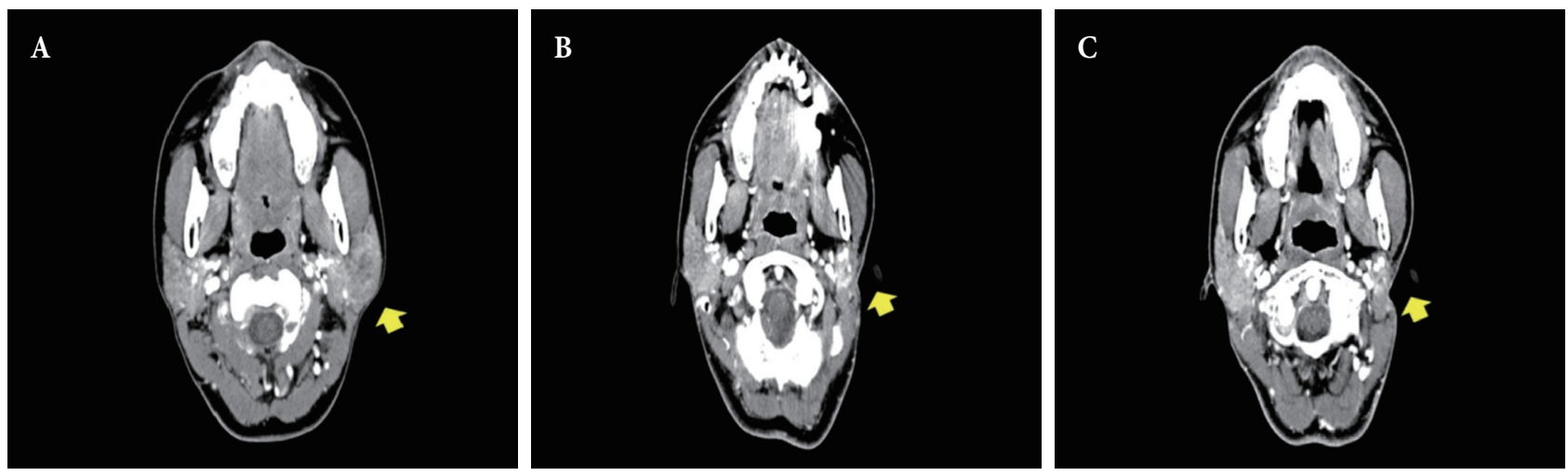

Fig. 3. A 41-year-old female patient with left parotid tumor who underwent parotid duct sacrificing superficial parotidectomy. (A) Preoperative computed tomography (CT) finding, (B) postoperative CT finding in 1 year, (C) postoperative CT finding in 2 years. Marked depression was noticed postoperatively (arrows).

analysis should be done on larger groups and longer follow-up periods in future study.

\section{CONFLICT OF INTEREST}

No potential conflict of interest relevant to this article was reported.

\section{PATIENT CONSENT}

The patients provided written informed consent for the publication and the use of their images.

\section{REFERENCES}

1. Gooden EA, Gullane PJ, Irish J, Katz M, Carroll C. Role of the sternocleidomastoid muscle flap preventing Frey's syndrome and maintaining facial contour following superficial parotidectomy. J Otolaryngol 2001;30:98-101

2. Ogreden S, Ruzgar S, Alimoglu Y, Eroglu S, Taskin U, Oktay MF. Comparison of Frey syndrome rates following superficial parotidec- tomy and partial superficial parotidectomy for pleomorphic adenoma. J Craniofac Surg 2016;27:e469-71.

3. Papadogeorgakis N, Skouteris CA, Mylonas AI, Angelopoulos AP. Superficial parotidectomy: technical modifications based on tumour characteristics. J Craniomaxillofac Surg 2004;32:350-3.

4. Wennmo C, Spandow O, Emgard P, Krouthen B. Pleomorphic adenomas of the parotid gland: superficial parotidectomy or limited excision? J Laryngol Otol 1988;102:603-5.

5. Lam KH, Wei WI, Lau WF. Tumours of the parotid: the value of clinical assessment. Aust N Z J Surg 1986;56:325-9.

6. Richards AT, Digges N, Norton NS, Quinn TH, Say P, Galer C, et al. Surgical anatomy of the parotid duct with emphasis on the major tributaries forming the duct and the relationship of the facial nerve to the duct. Clin Anat 2004;17:463-7.

7. Davis RA, Anson BJ, Budinger JM, Kurth LR. Surgical anatomy of the facial nerve and parotid gland based upon a study of 350 cervicofacial halves. Surg Gynecol Obstet 1956;102:385-412.

8. Yoo YM, Lee JS, Park MC, Kim C, Seo SJ, Lee IJ. Dermofat graft after superficial parotidectomy via a modified face-lift incision to prevent Frey syndrome and depressed deformity. J Craniofac Surg 2011;22:1021-3

9. Chang JW, Leem SS, Choi HJ, Lee JH. Modified functional superficial parotidectomy with ligation of the major branch of the parotid duct extending to the superficial lobe. Ann Plast Surg 2017;78:507-10. 